\title{
Rhabdomyoma of the pleura: a case report
}

\author{
Wei Wang ${ }^{1 \#}$, Ying-Ying Gü ${ }^{2 \#}$ Yu-Hong Dong ${ }^{3}$, Ji-Gang Wang ${ }^{1}$, Dong-Liang Lin ${ }^{1}$, Cong-Juan Luo ${ }^{4}$, \\ Li Zhang ${ }^{1}$, Xiao-Bin Ji ${ }^{1}$, Yan Wang ${ }^{1}$
}

${ }^{1}$ Department of Pathology, the Affiliated Hospital of Qingdao University, Qingdao 266555, China; ${ }^{2}$ The State Key Laboratory of Respiratory Disease, Guangzhou Institute of Respiratory Health, the First Affiliated Hospital, Guangzhou Medical University, Guangzhou 510120, China; ${ }^{3}$ Department of Liver Disease, the Sixth People's Hospital of Qingdao, Qingdao 266555, China; ${ }^{4}$ Department of Nephrology, the Affiliated Hospital of Qingdao University, Qingdao 266555, China

\#These authors contributed equally to this work.

Correspondence to: Yan Wang. Department of Pathology, the Affiliated Hospital of Qingdao University, No.1677, Wutaishan Road, Huangdao District, Qingdao 266555, China. Email: wylovegod@163.com.

Submitted May 22, 2018. Accepted for publication Nov 08, 2018.

doi: $10.21037 /$ jtd.2018.11.88

View this article at: http://dx.doi.org/10.21037/jtd.2018.11.88

\section{Introduction}

Rhabdomyoma is a rare benign soft tissue tumor deriving from striated muscle. It can be classified generally into two subtypes according to the location: cardiac rhabdomyoma and extracardiac rhabdomyoma. Cardiac rhabdomyoma manifests as well-defined solitary or multifocal nodules in heart consisting myocardial-like tumor cells with rich cytoplasm, which is more common in kids and associated with tuberous sclerosis (1). Extracardiac rhabdomyoma can be divided into three groups according to morphological features (adult, fetal, and genital types). The adult and fetal types mainly occur in the head and neck region, while the genital type usually occurs in the genital tract.

To the best of our knowledge, rhabdomyoma is rarely found in the respiratory system and the visceral pleura. Only one case of pulmonary rhabdomyoma has been reported in the English literature (2). It is difficult to make a correct pre-operative diagnosis. Herein, we report the first case of rhabdomyoma originating from visceral pleura.

\section{Case presentation}

A 66-year-old male presented to our thoracic surgery clinic complaining of chest pain for more than 6 months. $\mathrm{He}$ had a history of femoral head necrosis under conservative treatment for more than 10 years. Chest computed tomography (CT) scan revealed a 5 -cm solid visceral pleura mass in the basal segment of the right lower lobe (Figure 1). No symptom or radiographic evidence hint the tuberous

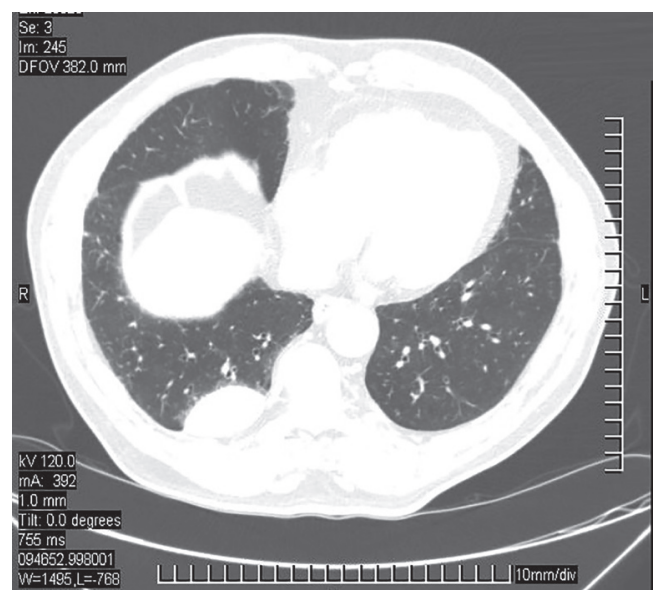

Figure 1 Tumor in the lower lobe of the right pleura.

sclerosis, a localized malignant mesothelioma could not be excluded.

Video-assisted thoracoscopic surgery was performed. The tumor was found in the visceral pleura in the basal segment of the right lower lobe with mild adhesion to the parietal pleura. It appeared as a solid mass about $5 \mathrm{~cm} \times$ $5 \mathrm{~cm} \times 3 \mathrm{~cm}$ with a well-defined boundary. Macroscopically, it showed a brown color with a jelly-like cut-surface.

Microscopically, the tumor was well-defined and did not invade the surrounding lung parenchyma (Figure $2 A$ ). Tumor cells were haphazardly arranged and presented various morphology including elongated, round, oval and polygonal with bright eosinophilic cytoplasm. An 


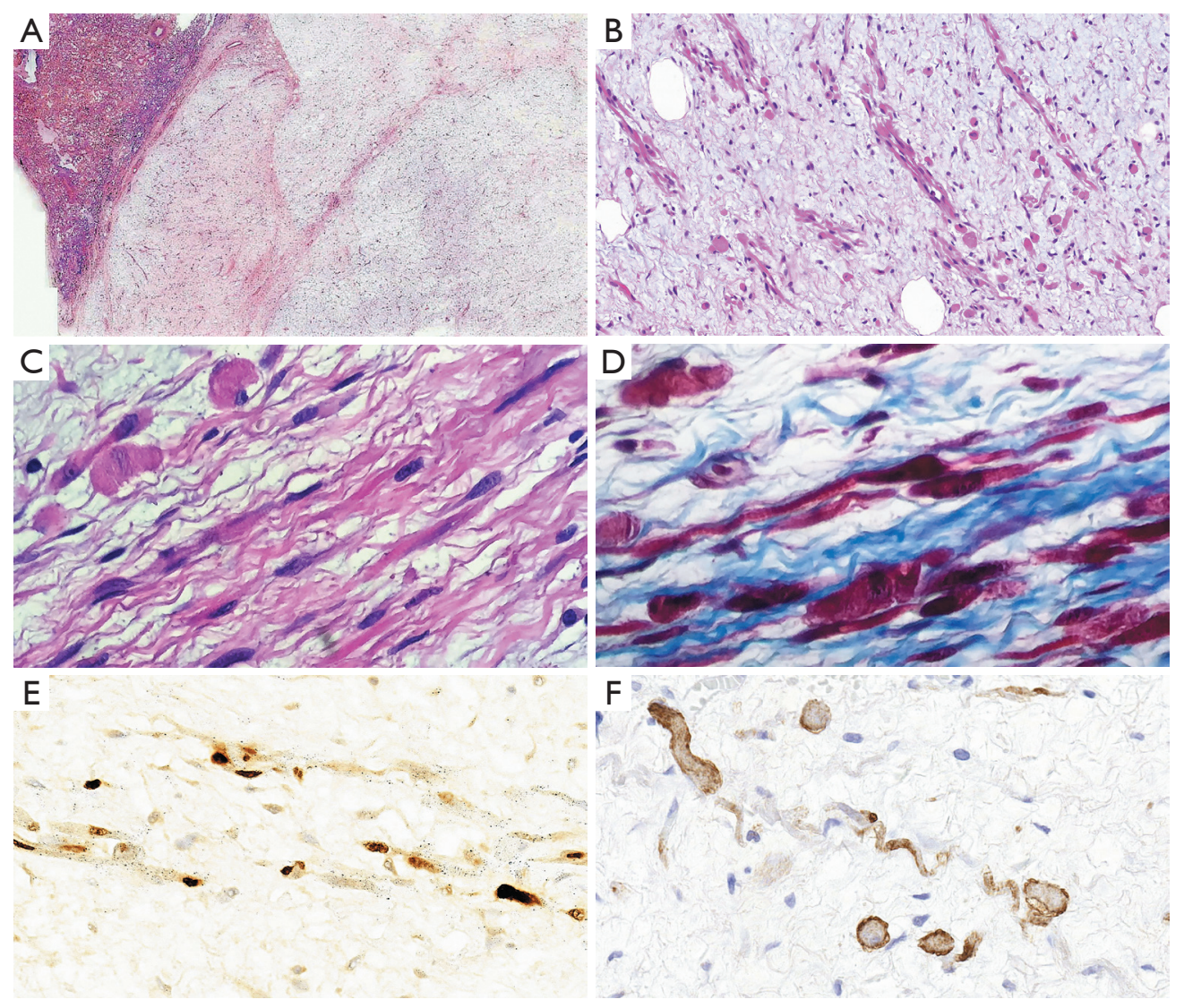

Figure 2 Histopathology evaluation of the pleural rhabdomyoma. (A) The tumor is attached to the pleura (HE, $\times 20$ original magnification); (B) haphazardly arranged muscle fibers in pleura, with an extensive mucinous degeneration of the mesenchyme (HE, $\times 40$ original magnification); (C) individual skeletal muscle differentiation with cross-striation is noted. Note the elongated, round, oval, to polygonal cells with bright eosinophilic cytoplasm and an eccentric nucleus (HE, $\times 200$ original magnification); (D) note the highlighted cross striation by Masson staining ( $\times 400$ original magnification); (E) IHC stain for myoD1 is positive ( $\times 400$ original magnification); (F) IHC stain for desmin is positive ( $\times 400$ original magnification).

eccentric nucleus was observed in some cells (Figure 2B). Cross-striations, the specific symbol of skeletal muscle differentiation, were observed in some tumor cells (Figure 2C). Atypia was not apparent. Irregular mitosis or necrosis were not observed. The mesenchyme appeared to be extensive mucous. Masson's trichrome staining highlighted the cross-striation in some tumor cells (Figure 2D). Immunohistochemistry for MyoD1 and Desmin, the markers of skeletal muscle differentiation, were positive (Figure 2E,F). All the above supported the diagnosis of a fetal rhabdomyoma. The chest pain had alleviated gradually after operation. The patient was uneventful till now, 30 months after operation.

\section{Discussion}

Rhabdomyoma, named by Zenker (3) in 1864 , is an exceedingly rare benign tumor that exhibits mature skeletal muscle differentiation. Among different rhabdomyoma subtypes, fetal rhabdomyoma is less common than the adult type. The average onset age of fetal rhabdomyoma is 4 years and it occurs more commonly in males than females (ratio 2.4:1) (4). The lesion usually presents as a smooth, movable, solitary (but occasionally multifocal), round or polypoid nodule in the head and neck region, or as a circumscribed intramuscular mass in the tonsil, epididymis, brain, tongue, sublingual region, lips, cheek, orbit, or 
submandibular region that is neither tender nor painful (5-8). In the presented case, both the onset age (66 years) and the location (the visceral pleura) are uncommon.

One of the main histological differential diagnoses of the fetal rhabdomyoma is the granular cell tumor, which consists mainly of smaller cells without cross-striations and shows diffuse S-100 expression and negative Desmin. The present case is positive for Desmin and negative for S-100, which excludes the diagnosis of granular cell tumor. Another differential diagnosis is embryonal rhabdomyosarcoma, which is characterized by cellular atypia, irregular mitosis and necrosis. None of these features was present in our case.

The origin of the striated muscle fibers is a fascinating mystery, as we all known, there is no striated muscle fibers in the pleura. Here, we have two bold inferences: First, this case is closely related to the parietal pleura, and there are striated muscles in the chest wall, it is reasonable to assume that the striated muscles are developed from misplaced striated muscle tissue of the chest wall. Second, in the formation of the primitive body cavity, pleural cavity develops from coelomic duct, while the latter is developed from the mesoderm, where primitive mesenchymal cells exist. There is a possibility that striated muscles are developed from metaplastic transformation of pluripotential mesenchymal cells in the pleural cavity. However, both the inferences above need further research and more evidence to support.

Rhabdomyoma can undergo recurrence, while malignancy transformation has not been described in the literature. A previous study showed that the local recurrence rate was $42 \%$ due to incomplete tumor resection, which occurred ranging between 2 and 11 years after diagnosis. The preferable treatment is complete resection with a clear surgical margin (9).

\section{Conclusions}

In conclusion, we report a rare case of pleural fetal rhabdomyoma. The accurate diagnosis was a challenge both for clinicians and for pathologists. The rhabdomyoma should not be excluded when a well-defined nodule is found in the visceral pleura.

\section{Acknowledgements}

None.

\section{Footnote}

Conflicts of Interest: The authors have no conflicts of interest to declare.

Informed Consent: Written informed consent was obtained from the patient for publication of this case report and any accompanying images.

\section{References}

1. Fenoglio JJ Jr, MCAllister HA Jr, Ferrans VJ. Cardiac rhabdomyoma: a clinicopathologic and electron microscopic study. Am J Cardiol 1976;38:241-51.

2. Lee BM. Primary rhabdomyoma of the lung. Int Surg 1975;60:229-30.

3. Arola DD, Bajaj D, Romberg E, et al. Comments on: "Hertzian contact response of dentin with loading rate and orientation" by N.R. da Silva, F. Lalani, P.G. Coelho, E.A. Clark, C.A. de Oliveira Fernandes, V.P. Thompson [Arch. Oral Biol. 53 (2008) 729-735]. Arch Oral Biol 2009;54:1125-7.

4. Kapadia SB, Meis JM, Frisman DM, et al. Fetal rhabdomyoma of the head and neck: a clinicopathologic and immunophenotypic study of 24 cases. Hum Pathol 1993;24:754-65.

5. Wang CP, Chang YH, Chang YT. Fetal Rhabdomyoma of the Right Tonsil with Polyp-Like Appearance. Case Rep Otolaryngol 2015;2015:713278.

6. Han Y, Qiu XS, Li QC, et al. Epididymis rhabdomyoma: a case report and literature review. Diagn Pathol 2012;7:47.

7. Santiago-Dieppa DR, Zhou T, Jones KA, et al. Intracranial Rhabdomyoma: Case Report and Review of the Literature. Cureus 2016;8:e593.

8. Favia G, Lo Muzio L, Serpico R, et al. Rhabdomyoma of the head and neck: clinicopathologic features of two cases. Head Neck 2003;25:700-4.

9. Kapadia SB, Meis JM, Frisman DM, et al. Adult rhabdomyoma of the head and neck: a clinicopathologic and immunophenotypic study. Hum Pathol 1993;24:608-17.

Cite this article as: Wang W, Gu YY, Dong YH, Wang JG, Lin DL, Luo CJ, Zhang L, Ji XB, Wang Y. Rhabdomyoma of the pleura: a case report. J Thorac Dis 2018;10(12):E793-E795. doi: $10.21037 /$ jtd.2018.11.88 